\title{
Nutrition Information in Oncology - Extending the Electronic Patient-Record Data Set
}

\author{
Priscila A. Maranhão ${ }^{1}$ (1D * Ana Margarida Pereira ${ }^{1,2}$ - Conceição Calhau ${ }^{1,3}$ • Paula Ravasco ${ }^{4,5,6}$ • Federico Bozzetti ${ }^{7}$. \\ Alessandro Laviano $^{8} \cdot$ Liz Isenring $^{9} \cdot$ Elisa V. $^{\text {Bandera }}{ }^{10}$ • Maureen B. Huhmann ${ }^{11}$ • Pedro Vieira-Marques ${ }^{1}$. \\ Ricardo J. Cruz-Correia ${ }^{1}$
}

Received: 12 March 2020 / Accepted: 25 August 2020 / Published online: 28 September 2020

(C) Springer Science+Business Media, LLC, part of Springer Nature 2020

\begin{abstract}
Electronic health records (EHRs) present extensive patient information and may be used as a tool to improve health care. However, the oncology context presents a complex content that increases the difficulties of EHR application. This study aimed at developing openEHR-archetypes representing clinical concepts in cancer nutrition-care, as well as to develop an openEHRtemplate including the aforementioned archetypes. The study involved the following stages: 1) a thorough literature review, followed by an expert's (nutrition guideline authors) survey, aiming to identify the main statements of published clinical guidelines on nutrition in cancer patients that were not included on the Clinical Knowledge Manager (CKM) repository; 2) modelling of the archetypes using the Ocean Archetype Software and submission to the CKM repository; 3) creating an example template with Template Designer; and 4) automatic conversion of the openEHR-template into a readily usable EHR using VCIntegrator. The clinical concepts (among 17 clinical concepts not yet available in the CKM repository) chosen for further development were: body composition, diet plan, dietary nutrients, dietary supplements, dietary intake assessment, and Malnutrition Screening Tool (MST). So far, four archetypes were accepted for review in the CKM repository and a template was created and converted into an EHR. This study designed new openEHR-archetypes for nutrition management in cancer patients. These archetypes can be included in EHR. Future studies are needed to assess their applicability in other areas and their practical impact on data quality, system interoperability and, ultimately, on clinical practice and research.
\end{abstract}

Keywords Electronic health records $\cdot$ Nutrition $\cdot$ Clinical practice guideline $\cdot$ openEHR $\cdot$ Oncology

This article is part of the Topical Collection on Patient Facing Systems

Electronic supplementary material The online version of this article (https://doi.org/10.1007/s10916-020-01649-9) contains supplementary material, which is available to authorized users.

Priscila A. Maranhão

pmaranhao@med.up.pt

1 Faculty of Medicine, Center for Research in Health Technologies and Information Systems (CINTESIS), University of Porto,

Porto, Portugal

2 CUF-Porto Instituto and Hospital, Porto, Portugal

3 Nova Medical School, Nova University Lisbon, Lisbon, Portugal

4 University Hospital of Santa Maria, Lisbon, Portugal

5 University of Lisbon, 1649-028 Lisbon, Portugal
6 Centre for Interdisciplinary Research in Health (CIIS) Portuguese Catholic - University, 1649-023 Lisbon, Portugal

7 Faculty of Medicine, University of Milan, Milan, Italy

8 Department of Clinical Medicine, Sapienza University, Rome, Italy

9 Bond University, Gold Coast, Australia

10 Rutgers Cancer Institute of New Jersey, Robert Wood Johnson Medical School, New Brunswick, NJ, USA

11 Rutgers, The State University of New Jersey Department of Nutrition Sciences, Newark, NJ, USA 


\section{Introduction}

Electronic Health Record (EHR) systems contribute to the provision of efficient, high-quality and integrated healthcare [1]. However, previous studies showed that EHRs have issues, especially regarding the effective sharing of complete patient data between heterogeneous information technology (IT) systems [2-4]. These interoperability issues, that lead to incompatible data exchange, hindering health care and research, need still heavy improvement and research [5].

In order to solve these problems, several standards have been proposed to represent and exchange electronic health data [6-9]. Currently, the top standard is HL7, which provides a complete framework for the exchange, integration, sharing, and retrieval of electronic health information [9]. Regarding interoperability, Fast Healthcare Interoperability Resources (FHIR) is an HL7 specification for the electronic exchange of healthcare information [10]. FHIR consists of two main parts - a content model (for clinical or administrative contents of the health records) in the form of 'resources', and a specification for the exchange of these resources [9]. The data are represented as knowledge artifacts that are structured, computable, and shareable representations of clinical knowledge [11]. Although widely spread, FHIR presents some drawbacks, which are addressed by other standards.

openEHR is another standard that comprises a set of open specifications for EHR architecture, aiming at enabling semantic health information interoperability between EHR systems. openEHR consists of a two-level framework: the reference model and the archetype model [12]. The latter is the most appealing aspect of openEHR, as it provides the structures for facilitating adequate data collection, data sharing and fine-grained EHR querying [13] and for allowing a structured and complete representation of the clinical models as suggested in disease guidelines. The main approach difference between openEHR and FHIR is that archetypes are maximal datasets, expected to represent all the clinical content, while FHIR resources only contain the most commonly used clinical information, but can be extended with additional items for a specific use case. Thus, openEHR archetype development is more prone to be performed by or with the direct involvement of healthcare professionals, who present the knowledge that is necessary to develop these maximal clinical content datasets [10, 14].

Promoting the interchangeability of patient health information among different medical care providers and services, is crucial, namely in oncology. In fact, cancer has been considered, in many cases, a chronic disease [15]. The number of patients who live longer with the disease has increased [16], stressing the care support on the healthcare systems. Furthermore, EHR has the potential to enhance oncological disease diagnosis and management, by allowing quicker pathology identification and, therefore, helping to prevent diagnostic delay, through alerts, reminders, personalized suggestions from clinical guidelines, among others [17-19]. Nevertheless, many critical data in oncology, some crucial to treatment decisions, such as biomarkers, disease staging, including metastasis location, and disease progression [20], do not have EHR support, while over half of the cancer variables are collected in an incomplete and unstructured form [21]. The potential importance of structured EHR data collection and interoperability is supported by the relevance given to the development of a guide with minimal common oncology Data Elements (mCODETM), implemented in HL7 FHIR. mCODETM is an initiative intended to assemble a core set of structured data elements for oncology EHRs and is a step towards capturing research-quality data from the treatment of all cancer patients. Furthermore, a multi-stakeholder community called CodeX — the Common Oncology Data Elements eXtensions - , is forming within the FHIR accelerator program. CodeX will expand around mCODE ${ }^{\mathrm{TM}}$ to encompass additional use cases, accelerating opportunities to create a learning health system based on interoperable data and improved patient care [22]. Also, in openEHR a few initiatives have been underway, namely targeting the development of oncology-specific archetypes. Chen et al. (2009), for example, modeled chemotherapy guidelines, and, by reusing several existing archetypes and authoring new archetypes and templates, were able to represent a lymphoma treatment guideline into an openEHRtemplate [23]. The modelled archetypes, however, were not submitted nor peer-reviewed by the Clinical Knowledge Manager (CKM), an international archetype repository. The openEHR-CKM has an ongoing project on cancer reporting; however, it is still very limited [24].

Nutrition management is particularly relevant regarding the care of cancer patients $[25,26]$. It is known that patients with cancer often present nutritional deficiencies, malnutrition and consequently weight loss up to $40 \%$, depending on the type of cancer $[27,28]$. These numbers led to the development of specific guidelines to support better nutrition care for cancer patients. Nonetheless, current EHRs are not adapted to the specificities of nutrition care, namely with a lack of specific documentation on nutrition safety and effectiveness [29]. Despite the need to enhance EHR in oncology and nutrition contexts, studies approaching strategies to improve these issues are very scarce. Developing specific templates to record clinical data suggested in guidelines for nutrition in cancer care can support a better access to clinical data and ultimately lead to better patient care, especially if 
using a standard that allows structured data representation and interoperability.

The present study aimed to develop openEHR-archetypes capable of representing clinical concepts in nutrition for cancer care, as well as to develop an openEHR-template using the developed archetypes.

\section{Material and methods}

This study was performed using the approach suggested by Bacelar-Silva et al. [30] Braun et al. [31] and Moner et al. [32], comprising the following sequential steps (Fig. 1).
Fig. 1 Flowchart of the openEHR based approach for clinical oncology data
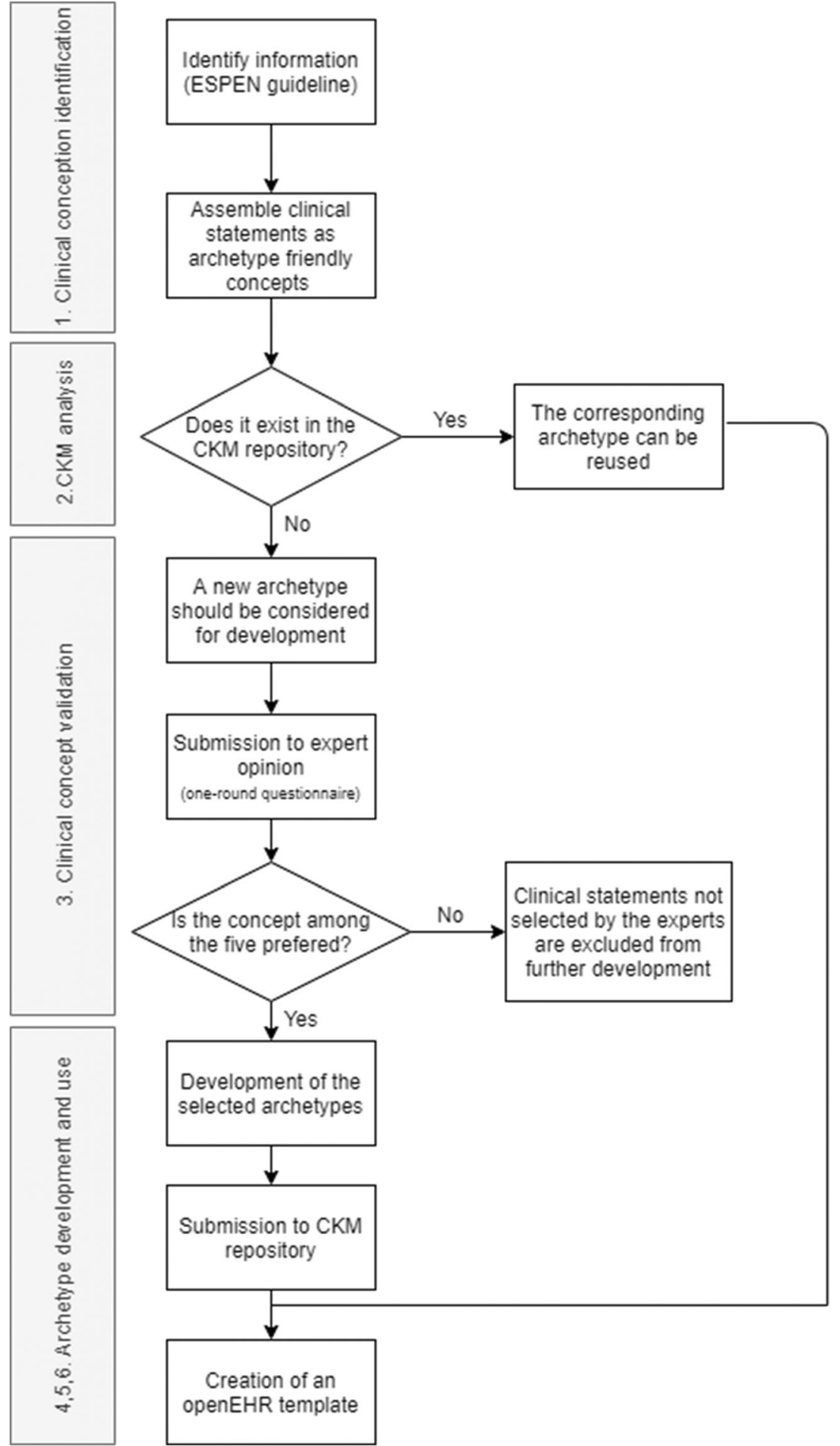

Identify information

(ESPEN guideline)

Assemble clinica

statements as

concepts

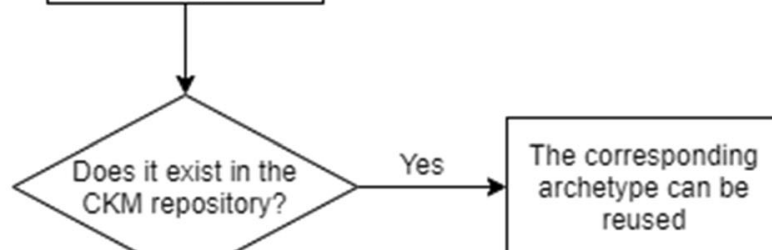

No

A new archetype

should be considered

for development

Submission to expert opinion

(one-round questionnaire)

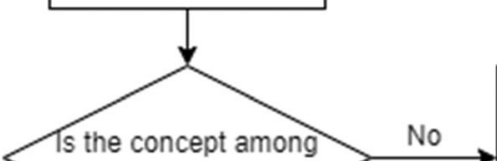

Clinical statements not selected by the experts

are excluded from

further development archetype friendly 


\section{Clinical concepts identification}

The first task was to identify the clinical statements in the European Society for Clinical Nutrition and Metabolism (ESPEN) guideline on nutrition for cancer patients [26]. This guideline was chosen because it is the most up to date in supporting nutrition management in cancer patients. The task was performed by three authors, one nutritionist, and two physicians, that independently identified and listed the clinical statements (e.g. body composition, body mass index) within the ESPEN guidelines. This selection was performed considering the nature of the statements and their objectives in clinical practice [31]. Thereafter, the identified clinical statements were organized and structured as archetype-friendly concepts, as described by Bacelar-Silva et al. [30] and Maranhão et al. [33].

After reviewing the ESPEN guidelines on nutrition for cancer patients, the authors identified 71 clinical statements and organized them into 23 archetype-friendly concepts (Table 1).

Table 1 Clinical statements, their respective archetype-friendly concepts and CKM status

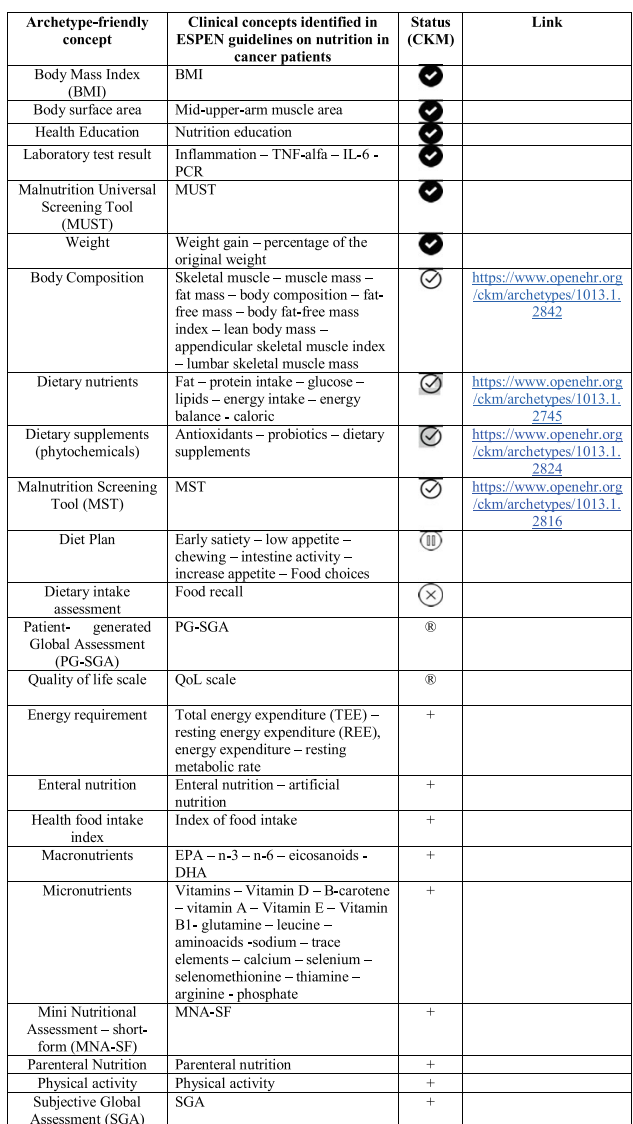

chetype existing in CKM; @ ( new archetypes approved by CKM; (iil new archetypes in review by $\mathrm{CKM} ; \otimes$ not approved by $\mathrm{CKM}$; ${ }^{\circledR}$ copyrighted instrument; + clinical statements identified in ESPEN guidelines, but not voted as relevant by nutrition experts.
Clinical Knowledge Manager (CKM) analysis

Subsequently, a CKM analysis was performed. CKM is an official archetype repository adopted to rule the knowledge in an international domain [24]. CKM repositories, namely international (International CKM - http://www.openehr.org/ $\mathrm{ckm} /$ ), Australia (NEHTA - http://dcm.nehta.org.au/ckm/) and United Kingdom (NHS - http://ckm.apperta.org/ckm/) were used to search for the concepts listed by the authors in order to avoid the development of duplicate archetypes. The search was executed using the name of the concept and the core-data items. New archetypes were considered for development when the concepts were not already available in the searched CKM repositories (Table 1).

\section{Clinical concept validation}

Subsequently, clinical-concept validation was performed by a panel of multidisciplinary experts in this area (e.g. nutritionists and physicians experts in nutrition science), using a one-round questionnaire. The study questionnaire was developed by a multidisciplinary team including a nutritionist and a physician. The clinical statements identified in the previous step were included in alphabetic order; those that were already present in the CKM ( $n=6)$ were excluded from the questionnaire. A pilot test was performed with healthcare professional volunteers $(n=5)$ aiming to detect errors and collect opinions about the survey questions. The questionnaire was considered easy to understand and no change was suggested.

The experts are co-authors of the main guidelines on nutrition, namely from the ESPEN, American Cancer Society (ACS), and American Society for Parenteral and Enteral Nutrition (ASPEN), and were contacted individually by email to fill a questionnaire comprising one question on the relevance of the archetype-concepts chosen by the paper authors ("Which are the five most relevant clinical statements to be registered in cancer patient records regarding nutritional information?"). The experts were asked to order their preferred 5 archetypes-concepts from 1, the most important, to 5, the least important. Additionally, the professionals could give their opinion on the relevance of each clinical concept, add other clinical statements or mark "none", in case they did not consider any clinical statement relevant.

\section{Archetype modelling in openEHR}

Subsequently, the selected concepts were modelled as openEHR archetypes. Archetype modelling has been widely applied in previous studies [30, 32, 34]. The concepts were discussed by the authors and relevant items were identified from books, journal articles, and guidelines. Afterwards, the archetype data was structured considering that each data is internally coded by a term code (at-code), which provides 
identity to the data nodes of the archetypes (i.e. to "name" the data) and value sets for leaf attributes [35].

The Ocean Archetype Editor software was used to model the archetype concepts [36]. The Archetype Editor provides graphical user interfaces that support openEHR-archetype creation and edition [31]. All archetypes were modelled in English.

\section{CKM submission for review}

After development, the archetypes were submitted to the CKM review-board through the openEHR.org platform. This platform works as an archetype repository with a review control-system. If the archetype is approved by CKM reviewers, it will have passed through the rigorous review of an international clinical community.

\section{openEHR-template and user interface}

The final step was the creation of an example openEHRtemplate, including both archetypes conceived in this study and some previously developed ones. The Ocean Template Design software was used to generate the template, which was then tested using the VCIntegrator software [37]. VCIntegrator allowed the automatic conversion of openEHR-template into a readily usable EHR, as mentioned by Sousa, E. et al. [38] and Trindade-Vilaça et al. [39].

\section{Statistical analysis}

Descriptive statistics were used to summarize the voting process by the nutrition experts. The score was calculated considering the relevance (1st-5th) stipulated by the experts, using the following correspondence: $1 \mathrm{st}=5$ points, $2 \mathrm{nd}=4$ points, $3 \mathrm{rd}=3$ points; 4 th $=2$ points and 5 th $=1$ point. Individual scores were summed to give the final score to each archetype-concept. Ties were settled by the number of votes; however, if the ties remained after these two classification systems, the last tied archetypes were considered to be modeled.

\section{Results}

A total of 34 nutrition experts were contacted, and 7 answered the questionnaire ( 3 nutritionists and 4 physicians). The first 5 concepts were: body composition, Patient-Generated Subjective Global Assessment (PG-SGA), diet plan, quality of life scale (QoLs) and dietary nutrients (Table 2). However, PG-SGA and QoL scale are copyrighted instruments and cannot be directly modelled as openEHR-archetypes without formal authorization from the authors; these concepts were replaced by the following most voted archetypes, dietary supplements
Table 2 Summary of the voting process by the experts and scores attained by each concept

\begin{tabular}{llll}
\hline Archetype-friendly concept & $\begin{array}{l}\text { Votes } \\
\text { (n) }\end{array}$ & Score & Rank \\
\hline Body composition & 6 & 18 & $\mathbf{1}$ st \\
Patient-Generated Subjective Global Assessment & 3 & 15 & $\mathbf{2 n d}$ \\
$\quad$ (PG-SGA) & & & \\
Diet plan (e.g. satiety, chewing, appetite, etc.) & 3 & 9 & 3rd \\
Quality of life scale (QoLs) & 2 & 9 & $\mathbf{4 t h}$ \\
Dietary nutrients (e.g. protein, glucose) & 2 & 8 & $\mathbf{5 t h}$ \\
Dietary supplements (e.g. phytochemicals) & 3 & 7 & $\mathbf{6 t h}$ \\
Dietary intake assessment (e.g.: food diary, & 2 & 6 & $\mathbf{7 t h}$ \\
$\quad$ R24h, etc.) & 2 & 6 & $\mathbf{7 t h}$ \\
Malnutrition Screening Tool (MST) & 3 & 5 & 8 th \\
Physical Activity & 1 & 5 & 9 th \\
Health food intake index & 2 & 4 & 10 th \\
Macronutrients & 2 & 4 & 10 th \\
Micronutrients & 1 & 4 & 11 th \\
Energy requirement & 1 & 4 & 11 th \\
Subjective Global Assessment (SGA) & 1 & 3 & 12 th \\
Enteral Nutrition & 1 & 1 & 13 th \\
Parenteral Nutrition & 0 & 0 & 14 th \\
Mini Nutritional Assessment Short Form & $1^{\text {a }}$ & - & - \\
$\quad$ MNA-SF) & - & - & - \\
Other & & & \\
None & & & \\
\hline
\end{tabular}

The score was calculated considering the relevance ( $1 \mathrm{st}-5$ th) stipulated by the experts, using the following correspondence: 1 th $=5$ points, 2 th $=4$ points, 3 th $=3$ points; 4 th $=2$ points and 5 th $=1$ point. To tiebreak, the number of votes was considered. ${ }^{\text {a }}$ One expert suggested, additionally, "Nutrition impact on symptom profile (e.g.: nausea, etc.)

(phytochemicals) (6th), and dietary intake assessment and malnutrition screening tool (MST), tied in the 7th place.

After successful development, these 6 archetypes were submitted to the International CKM repository, and 4 (body composition, dietary nutrients, dietary supplements (phytochemicals) and MST) were approved by the CKM editors. Links to the mind map representations of the accepted archetypes are presented in Table 1. The archetype "diet plan", which intends to record information about an individual's diet, is still under review by the CKM editors. The archetype "dietary intake assessment" was not approved, since, according to the CKM editor, it comprises many concepts (e.g. food frequency questionnaire, 24-h diet recall, among others) that should be represented independently.

All created archetypes are available at https://github.com/ priscilamaranhao/oncology-archetypes ODISSEIA.git

The example openEHR-template was successfully created (Fig. 2) and was further represented in the user interface built with VCIntegrator (Fig. 3). Figures with an enhanced and 


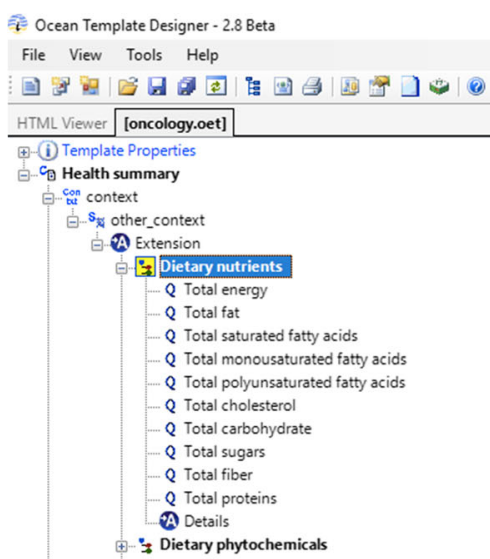

Fig. 2 Oncology template modelled from Template Design

wider detail of the example template and user interface are available as supplementary material.

\section{Discussion}

This study focused on describing the development process of openEHR archetype-based structures, used to represent cancer clinical statements proposing nutritional care. These archetypes can be used to improve EHR interoperability [40].

Some authors have suggested that EHRs have a strong potential to improve cancer outcome research [41] and patient care $[42,43]$. However, EHRs usage in oncology presents, currently, many challenges related to structural (e.g.: interoperability, incorporation of different data forms...), clinical (e.g.: codification, linking of departments and care continuity) and research-related issues (e.g.: missing data, generalizability, privacy) [41, 44]. The core set of cancer data elements established by $\mathrm{mCODE}$ and leveraged by CodeX are a recent attempt to solve the challenges related to the EHR storage of oncology data [44]. Nevertheless, in nutrition management for cancer patients, which is unanimously a relevant issue, no information is available about which variables should be stored.
Despite obtaining nutrition expert opinions, we cannot assert that we created the most important clinical concepts in the nutrition and cancer context, since some of the identified concepts were already represented in CKM (i.e. body mass index, weight, body surface area) and did not have their relevance assessed by the experts; moreover, only the ESPEN guidelines were considered in the identification of the clinical statements, and additional relevant concepts could be present in other guidelines. We believe that, since these are quite general concepts with direct applicability to other clinical areas, there was a great need for this development. Nevertheless, the "dietary intake assessment" archetype was not approved by CKM. They justified that this archetype contained multiple concepts and proposed a new archetype named "food diary". However, we understand that "dietary intake assessment" archetype should be only one concept, which intends to record the dietary assessment method (e.g., 24-h recall, food frequency questionnaire, among others), informing the dietitian on how was data obtained regarding food and beverages consumed on a given day.

In fact, openEHR is a relevant approach to model-driven development of EHR and stimulate clinicians to be an active part in the modelling process [45]. Kanas et al. described the importance of having medical professionals guiding the development of EHR systems as a way to improve the quality of

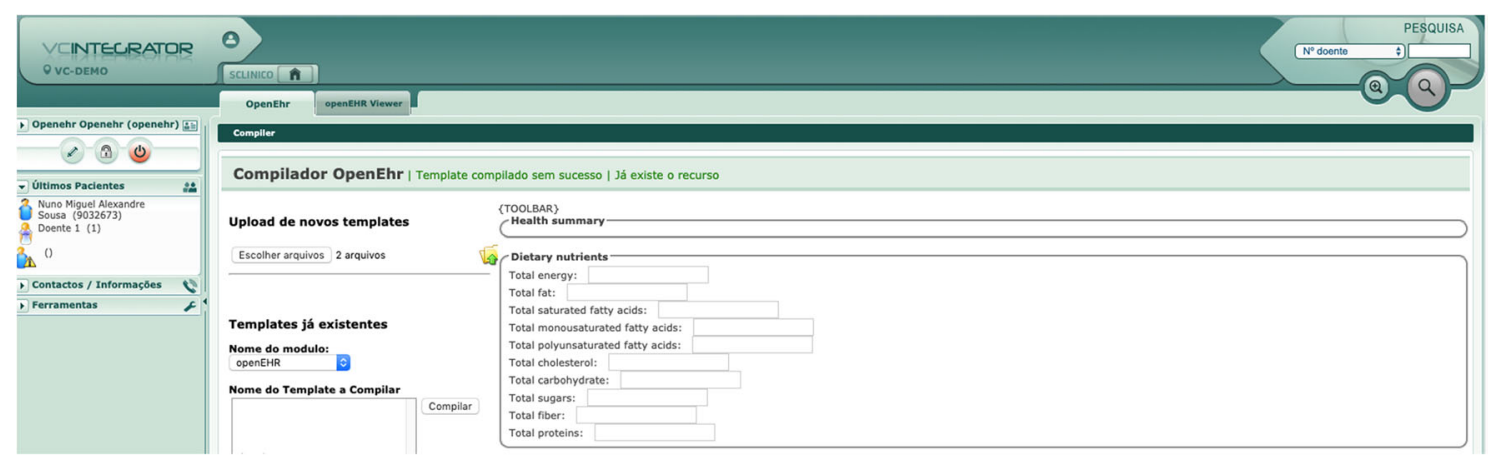

Fig. 3 View of the template after automatic conversion into an EHR, by VCIntegrator 
cancer care through oncology outcome research [42]. This is possible through openEHR standards, which allow that clinical statements are evenly modelled by physicians. As a result, openEHR-archetype modelling has been used in many studies to enhance EHR in specific clinical areas, such as nutrigenomics and multiple sclerosis functional assessments $[31,46]$. Nonetheless, despite these efforts, the CKM repository presents few archetypes to answer clinical management demands in some areas. Therefore, to make this approach more effective, there is a paramount need for more archetypes to be modelled, always respecting guidelines, published clinical research and expert opinions, to develop trustworthily and validated tools.

Nevertheless, copyrighted tools cannot be represented into the openEHR standard without a formal acceptance from the authors, agreeing, during the process, on a more open license. openEHR is an open industry specification, not compatible with copyright-related restrictions. In the present study, despite being considered an important tool by the experts, the most adequate oncology-specific QoL scales and PG-SGA presented copyrights and could not be directly designed as an openEHR-archetypes. This issue limits the modelling of some important tools (e.g. questionnaires, scales, etc) that, if modeled in openEHR, could provide better support to the improvement of cancer management.

To the best of our knowledge, this study is the first to develop openEHR-archetypes, approved by the CKM, and a template representation for the management of nutrition in cancer patients. openEHR archetypes modelling is a relevant tool not only for nutrition but also for many other issues in cancer management and treatment. In fact, despite the scarcity of oncology openEHR-archetypes available in CKM repository, some researchers have used them to model an EHR capable of identifying and improving breast cancer treatment processes and chemotherapy guideline uses [47]. Those studies applied openEHR-archetypes because they allowed the EHR to become more structured, which may improve the interoperability, contributing to better clinical care and research.

openEHR is optimised to provide a data platform with a focus on the persistence of data, and the better way to store medical information [48], allowing the creation of standards, to build information and interoperability solutions. There are more than 500 archetypes in different medical areas that were already developed, such as related to nutrition, COVID-19, genomics, obstetrics/gynaecology, and so on. Conversely, HL7 FHIR, despite being focused on interoperability and data as well, is optimized for exchange of data, through the provision of simple and easy to use REST API's. The basic building block of FHIR is a resource, which is used, for instance, to exchange clinical content, create care plans and diagnostic orders [48, 49].

Finally, we demonstrated an example of the conversion of a specific-template, built with openEHR-archetypes, into an
EHR form, which also supports the practical and borderless applicability of the developed openEHR-archetypes. Health professionals that download openEHR-archetypes from the CKM website can create a specific template for oncology treatment and automatically convert it in an EHR form, without the need for information technology (IT) professionals.

This study has some limitations. First, there are many available guidelines for nutrition management in cancer patients, but they were not reviewed in this study. However, we believe that the most significant clinical concepts in this area are similar among the different guidelines. In addition, nutrition is an extensive research field, and choosing the most relevant clinical statements is challenging. Nevertheless, the clinical concepts were selected for further development by nutrition experts (nutrition guidelines authors) in this area, supporting the relevance of the openEHR-archetypes that were modelled herein.

The choice of the concepts to develop was based on oneround questionnaire. Although we acknowledge that using a Delphi would be better, we believed that, considering the use of the questionnaire just as a way to support the selection of the first archetypes to develop (which would be the most important ones), and to decrease the nutrition expert burden, a one-round questionnaire, although limited, would give enough information. However, this methodology was further limited by the low response rate of $20 \%$. Digital surveys usually present a lower response rate than the paper counterpart [50], thus a low number of responses was expected. In fact, a $20 \%$ response rate is inline with the values reported by other studies using similar data collection methodologies [51]. Moreover, the focus on guideline authors limited the number of potentially contactable individuals, further reducing the total number of participants, but ensuring that those who answered were experts on the subject.

Subsequently, our future perspective is to model a complete set of clinical statements for nutrition care in oncology and test it in a real-life study.

\section{Conclusions}

The present study described the process to identify and to model openEHR-archetypes for nutrition management in oncology patients and produced an example openEHR-template and EHR user interface. These tools can be applied by health and IT professionals to enhance structured data collection and promote interoperability between EHR systems.

Acknowledgments The authors would like to acknowledge ODISSEIA Oncology Information System project (POCI-05-5762-FSE-039021), financed by the North Portugal Regional Operational Programme (NORTE 2020), under the PORTUGAL 2020 Partnership Agreement, and through the European Regional Development Fund (ERDF) and European Social Fund (ESF) respectively. 
Authors 'contributions PAM and AMP - contributed to study design, conceptualization, modelled the openEHR archetypes and wrote the original draft; CC, PR - contributed to conceptualization, elaborated the paper methodology and questionnaire and wrote the original draft; FB, AL, LI, $\mathrm{EB}, \mathrm{MH}$ - analyzed the ESPEN guideline and wrote the original draft and reviewed the paper; PVM, RJCC - conceptualized and reviewed the paper.

Availability of data and materials The archetypes generated during the current study are available at oncology-archetypes_ODISSEIA repository, [https://github.com/priscilamaranhao/oncology-archetypes ODISSEIA.git].

Funding North Portugal Regional Operational Programme (NORTE 2020), under the PORTUGAL 2020 Partnership Agreement, and through the European Regional Development Fund (ERDF) and European Social Fund (ESF) respectively.

\section{Compliance with ethical standards}

Conflict of interest The authors declared that there is no conflict of interest.

\section{References}

1. OECD (2010) Improving Health Sector Efficiency: The Role of information and communication technologies. doi:https://doi.org/ 10.1787/9789264084612-en

2. van der Bij S, Khan N, Ten Veen P, de Bakker DH, Verheij RA (2017) Improving the quality of EHR recording in primary care: a data quality feedback tool. J Am Med Inform Assoc 24 (1):81-87. doi:https://doi.org/10.1093/jamia/ocw054

3. King J, Patel V, Jamoom EW, Furukawa MF (2014) Clinical benefits of electronic health record use: national findings. Health Serv Res 49 (1 Pt 2):392-404. doi:https://doi.org/10.1111/1475-6773. 12135

4. Kruse CS, Stein A, Thomas H, Kaur H (2018) The use of Electronic Health Records to Support Population Health: A Systematic Review of the Literature. J Med Syst 42 (11):214. doi:https://doi. org/10.1007/s10916-018-1075-6

5. Jiang G, Evans J, Oniki TA, Coyle JF, Bain L, Huff SM, Kush RD, Chute CG (2015) Harmonization of detailed clinical models with clinical study data standards. Methods Inf Med 54 (1):65-74. doi: https://doi.org/10.3414/me13-02-0019

6. Reisman M (2017) EHRs: The Challenge of Making Electronic Data Usable and Interoperable. P t 42 (9):572-575

7. Beale T, Heard S (2007) Archetype Definitions and Principles.

8. International HL7. https://www.hl7.org/.

9. International HL7 Standards - Section 1c: FHIR®-Fast Health Interop Resources. https://www.hl7.org/implement/standards/ product section.cfm?section $=12$.

10. Bosca D, Moner D, Maldonado JA, Robles M (2015) Combining Archetypes with Fast Health Interoperability Resources in Futureproof Health Information Systems. Stud Health Technol Inform 210:180-184

11. FHIR HL7 (2019) Representing Knowledge Artifacts.

12. Beale T (2002) Constraint-based Domain Models for Future-proof Information Systems, Revision: 2.2.1. vol 4. Eleventh OOPSLA Workshop on Behavioral Semantics: Serving the Customer

13. Chen R, Klein GO, Sundvall E, Karlsson D, Ahlfeldt H (2009) Archetype-based conversion of EHR content models: pilot experience with a regional EHR system. BMC Med Inform Decis Mak 9:33. doi:https://doi.org/10.1186/1472-6947-9-33

14. Cardoso de Moraes JL, de Souza WL, Pires LF, do Prado AF (2016) A methodology based on openEHR archetypes and software agents for developing e-health applications reusing legacy systems. Comput Methods Programs Biomed 134:267-287. doi:https://doi. org/10.1016/j.cmpb.2016.07.013

15. Phillips JL, Currow DC (2010) Cancer as a chronic disease. Collegian 17 (2):47-50. doi:https://doi.org/10.1016/j.colegn.2010. 04.007

16. Bertucci F, Le Corroller-Soriano AG, Monneur-Miramon A, Moulin JF, Fluzin S, Maraninchi D, Gonçalves A (2019) Outpatient Cancer Care Delivery in the Context of E-Oncology: A French Perspective on "Cancer outside the Hospital Walls". Cancers (Basel) 11 (2). doi:https://doi.org/10.3390/ cancers 11020219

17. Macleod U, Mitchell ED, Burgess C, Macdonald S, Ramirez AJ (2009) Risk factors for delayed presentation and referral of symptomatic cancer: evidence for common cancers. Br J Cancer 101 Suppl 2 (Suppl 2):S92-s101. doi:https://doi.org/10.1038/sj.bjc. 6605398

18. Poon EG, Kachalia A, Puopolo AL, Gandhi TK, Studdert DM (2012) Cognitive errors and logistical breakdowns contributing to missed and delayed diagnoses of breast and colorectal cancers: a process analysis of closed malpractice claims. J Gen Intern Med 27 (11):1416-1423. doi:https://doi.org/10.1007/s11606-012-2107-4

19. Castaneda C, Nalley K, Mannion C, Bhattacharyya P, Blake P, Pecora A, Goy A, Suh KS (2015) Clinical decision support systems for improving diagnostic accuracy and achieving precision medicine. J Clin Bioinforma 5:4. doi:https://doi.org/10.1186/s13336015-0019-3

20. Berger ML, Curtis MD, Smith G, Harnett J, Abernethy AP (2016) Opportunities and challenges in leveraging electronic health record data in oncology. Future Oncol 12 (10):1261-1274. doi:https://doi. org/10.2217/fon-2015-0043

21. Raghavan P, Chen JL, Fosler-Lussier E, Lai AM (2014) How essential are unstructured clinical narratives and information fusion to clinical trial recruitment? AMIA Jt Summits Transl Sci Proc 2014: 218-223

22. International HL7 CodeX. https://www.hl7.org/codex/.

23. Chen R, Georgii-Hemming P, Ahlfeldt H (2009) Representing a chemotherapy guideline using openEHR and rules. Stud Health Technol Inform 150:653-657

24. openEHR Clinical Knowledge Manager. https://www.openehr.org/ $\mathrm{ckm} /$.

25. Virizuela JA, Camblor-Álvarez M, Luengo-Pérez LM, Grande E, Álvarez-Hernández J, Sendrós-Madroño MJ, Jiménez-Fonseca $\mathrm{P}$, Cervera-Peris M, Ocón-Bretón MJ (2018) Nutritional support and parenteral nutrition in cancer patients: an expert consensus report. Clin Transl Oncol 20 (5):619-629. doi:https://doi.org/10.1007/ s12094-017-1757-4

26. Arends J, Bachmann P, Baracos V, Barthelemy N, Bertz H, Bozzetti F, Fearon K, Hütterer E, Isenring E, Kaasa S, Krznaric Z, Laird B, Larsson M, Laviano A, Mühlebach S, Muscaritoli M, Oldervoll L, Ravasco P, Solheim T, Strasser F, de van der Schueren M, Preiser JC (2017) ESPEN guidelines on nutrition in cancer patients. Clin Nutr 36 (1):11-48. doi:https://doi.org/10.1016/j. clnu.2016.07.015

27. Rosania R, Chiapponi C, Malfertheiner P, Venerito M (2016) Nutrition in Patients with Gastric Cancer: An Update. Gastrointest Tumors 2 (4):178-187. doi:https://doi.org/10.1159/000445188

28. Steenhagen E, van Vulpen JK, van Hillegersberg R, May AM, Siersema PD (2017) Nutrition in peri-operative esophageal cancer management. Expert Rev Gastroenterol Hepatol 11 (7):663-672. doi:https://doi.org/10.1080/17474124.2017.1325320 
29. Vanek VW (2012) Providing nutrition support in the electronic health record era: the good, the bad, and the ugly. Nutr Clin Pract 27 (6):718-737. doi:https://doi.org/10.1177/0884533612463440

30. Bacelar-Silva GM, Chen R, Cruz-Correia RJ (2012) From clinical guideline to openEHR: converting JNC7 into archetypes and template. Paper presented at the XIII Congresso Brasileiro de Informática em Saúde

31. Braun M, Brandt AU, Schulz S, Boeker M (2014) Validating archetypes for the Multiple Sclerosis Functional Composite. BMC Med Inform Decis Mak 14:64. doi:https://doi.org/10.1186/14726947-14-64

32. Moner D, Maldonado JA, Robles M (2018) Archetype modeling methodology. J Biomed Inform 79:71-81. doi:https://doi.org/10. 1016/j.jbi.2018.02.003

33. Maranhão PA, Bacelar-Silva GM, Ferreira DNG, Calhau C, VieiraMarques P, Cruz-Correia RJ (2018) Nutrigenomic Information in the openEHR Data Set. Appl Clin Inform 9 (1):221-231. doi:https:// doi.org/10.1055/s-0038-1635115

34. Späth MB, Grimson J (2011) Applying the archetype approach to the database of a biobank information management system. Int $\mathrm{J}$ Med Inform 80 (3):205-226. doi:https://doi.org/10.1016/j.ijmedinf. 2010.11.002

35. openEHR Foundation (2015) openEHR architecture overview. https://specifications.openehr.org/releases/BASE/Release-1.0.3/ architecture_overview.html\#_binding_external_terminology_ codes to archetype codes.

36. openEHR Foundation An open domain-driven platform for developing flexible e-health systems. . https://openehr.org/.

37. VirtualCare VCIntegrator. http://icu-v08.gim.med.up.pt/.

38. Sousa E, Jesus T, Neves L, Costa M, Ribeiro FR, Metrôlho JC, Cruz-Correia R Integrating Medical Patient Data with Family Trees to Improve the Quality of Information. In, Berlin, Heidelberg, 2011. 5th International Conference on Practical Applications of Computational Biology \& Bioinformatics (PACBB 2011). Springer Berlin Heidelberg, pp. 17-22

39. Trindade-Vilaça C, Silva-Costa T, Cruz-Correia R Implementation of medical algorithms in the Breast Pathology module of VCIntegrator. In: 6th Iberian Conference on Information Systems and Technologies (CISTI 2011), 15-18 June 2011. pp 1-4 ,2011.

40. Min L, Tian Q, Lu X, An J, Duan H (2018) An openEHR based approach to improve the semantic interoperability of clinical data registry. BMC Med Inform Decis Mak 18 (Suppl 1):15. doi:https:// doi.org/10.1186/s12911-018-0596-8
41. Manion FJ, Harris MR, Buyuktur AG, Clark PM, An LC, Hanauer DA (2012) Leveraging EHR data for outcomes and comparative effectiveness research in oncology. Curr Oncol Rep 14 (6):494-501. doi:https://doi.org/10.1007/s11912-012-0272-6

42. Kanas G, Morimoto L, Mowat F, O'Malley C, Fryzek J, Nordyke R (2010) Use of electronic medical records in oncology outcomes research. Clinicoecon Outcomes Res 2:1-14. doi:https://doi.org/ $10.2147 /$ ceor.s8411

43. LeBlanc TW, Back AL, Danis M, Abernethy AP (2014) Electronic Health Records (EHRs) in the oncology clinic: how clinician interaction with EHRs can improve communication with the patient. J Oncol Pract 10 (5):317-321. doi:https://doi.org/10.1200/jop.2014. 001385

44. Ann Aschengrau, George R. Seage (2018) Essentials of epidemiology in public health. Jones \& Bartlett Learning, Burlington, MA.

45. Heather Leslie, Heard S (2006) Archetypes 101. Paper presented at the Health Informatics Society of Australia, Brunswick East, Vic,

46. Mascia C, Uva P, Leo S, Zanetti G (2018) OpenEHR modeling for genomics in clinical practice. Int J Med Inform 120:147-156. doi: https://doi.org/10.1016/j.ijmedinf.2018.10.007

47. Serna A, Gerrikagoitia JK, Huerga I, Zumalakarregi JA, Pijoan JI, Archetypes and ontologies to facilitate the breast cancer identification and treatment process.

48. Allen A., FHIR vs. openEHR., 2017 https://medium.com/@ alastairallen/fhir-vs-openehr-75a0a7c6e5a7.

49. FHIR HL7, Resource Explanation of benefit - Content. , 2019 https://www.hl7.org/fhir/explanationofbenefit.html.

50. Uhlig CE, Seitz B, Eter N, Promesberger J, Busse H (2014) Efficiencies of Internet-based digital and paper-based scientific surveys and the estimated costs and time for different-sized cohorts. PLoS One 9 (10):e108441. doi:https://doi.org/10.1371/journal. pone. 0108441

51. Ebert JF, Huibers L, Christensen B, Christensen MB (2018) Paperor Web-Based Questionnaire Invitations as a Method for Data Collection: Cross-Sectional Comparative Study of Differences in Response Rate, Completeness of Data, and Financial Cost. J Med Internet Res 20 (1):e24. doi:https://doi.org/10.2196/jmir.8353

Publisher's Note Springer Nature remains neutral with regard to jurisdictional claims in published maps and institutional affiliations. 